\title{
Amateurs and the Search for Supernovae
}

\author{
Stephen H. Lucas
}

14400 S. Kolin Ave., Midlothian, IL 60445, U.S.A.

The role of amateur astronomers in the search of extragalactic supernovae is emerging. Visual discoveries by John Bennett in 1968 and Gus Johnson in 1979 were isolated and surprising encounters, but are indicative of the possibilities. Between 1980 and the present, these possibilities have been well-tested by the Rev. Robert O. Evans, who has discovered 15 SN's and shares credit for a 16th. Rev. Evans' record was achieved through an organized approach to observation and assisted by the development of a unique observing regimen. Detection of $\mathrm{SN}$ is a formidable challenge, but the obvious conclusion is that amateurs may expect to attain more than marginal success. We approach this subject from the standpoint of visual observation, but are obliged to acknowledge the contemporaneous and successful application of photography as a search tool.

Steps that must be taken to improve the likelihood of visual discoveries depend on persistance, method, charts, and equipment, and it should be no surprise that motivation is a major guarantor of this undertaking. Amateur discoveries have contributed to the resurgence of general interest, especially within the past few years. They have contributed to the current flowering in the theoretical analysis of Type I SN, led by Profs. J. Craig Wheeler, David Branch, and others. Especially, two of these discoveries have led to the realization that there are two completely different classes of SN with a Type I light-curve. The new class is now called Type Ib, and the two SN 1983N and 1984L have been prototype examples of the class. The two brightest SN (1983N and 1986G) have also been used as probes of intergalactic space, and have resulted in the discovery of several "clouds" of gas, between the Milky Way group of galaxies, which had not been known before. There will be effects from this discovery in the study of quasars and other very distant objects.

With the proper organization, amateurs will be able to make a major contribution in deciding the rate of appearance of SN in the various types of galaxies. By collating and studying statistics of negative an positive search observations on many different galaxies, and over an extended period of time, we can know more clearly the average SN rate. An article recently submitted to Astrophysical Journal made a study of 50,000 visual observations of 1081 galaxies over a 5-year period. A sub-sample of 709 Shapley-Ames galaxies was used to help create homogeneity. Of the $29 \mathrm{SN}$ discovered world-wide in these galaxies during the 5 years, eleven were discovered by the visual observer, and 4 others were also seen, resulting in a total of 15 events 
falling within the scope of the search. Estimates of the SN rate were made on this basis. (Van den Bergh, et al. 1987)

The detection of these enormous stellar events represent one of the final bastions of scientific discovery left open to the amateur. Careful scrutiny is the price that has to be paid for such a project. Our group SUNSEARCH is not like most groups where the few do the work for the many. We are the few, and have elected, by intent, to participate in a tedious program of amateur astronomy. It certainly isn't the easiest specialization, but it can be rewarding; if not, then just making us better observers and perhaps making a new friend along the way. After all, isn't that what it's all about anyway? 